\title{
Autism research makes the leap to frogs
}

\author{
BY LAURA DATTARO
}

8 SEPTEMBER 2021

\section{Listen to this story:}

https://www.spectrumnews.org/wp-content/uploads/2021/09/audio-24121835-e34d-4d8bbb7b-a1810224b966-encodings.mp3

Earlier this year, researchers reported striking similarities in the functions of 10 autism genes: They all affect brain size and boost the number of immature neurons in the brain. The work raised hopes of finding common biological paths to autism.

Most studies on gene function are done in mice, but this work involved a tiny tropical frog, Xenopus tropicalis, that is native to West Africa.

These frogs boast a slew of traits that make them useful for autism research. Each adult can lay thousands of eggs, which develop into tadpoles in only a week. After the fertilized egg divides, each daughter cell and all of its progeny stay on their respective side. This leads to a perfectly bisected animal that can host a mutation on just one side of its body. Researchers can then watch how those cells develop early on: The tadpole's initial developmental stages occur outside of the mother's body. What's more, Xenopus can be induced to lay eggs any time, simply by exposing the frog to hormones.

The team edited each of 10 top autism genes in just one of the two original daughter cells in thousands of Xenopus eggs. As the tadpoles' brains developed, their two sides developed differently: The inserted mutations led to an atypically large or small cerebrum and an unusually large number of immature neurons, all of it clearly visible to researchers. "This [tadpole] is just out on his own, swimming," says study investigator Helen Willsey, a postdoctoral researcher in Matthew State's lab at the University of California, San Francisco. "We can look into the brain while it's alive and really watch all of brain development in a dish." 


\section{Spectrum | Autism Research News}

https://www.spectrumnews.org

Thanks to this transparency, research in frogs has led to foundational discoveries about the brain and nervous system, including that of the first neurotransmitter and of techniques to create human pluripotent stem cells. "Xenopus has been extraordinary in allowing us to watch the nervous system develop," says Hazel Sive, dean of the College of Science at Northeastern University in Boston, Massachusetts.

Researchers continue to come up with clever ways to study autism and neurodevelopment in the amphibians. One research team used frogs to show that deletions in the chromosomal region $3 q 29$, which are linked to autism, may accelerate the process of cell death, identifying a potential mechanism for the deletion's effects. In an unpublished study, another team found that knocking out the autism-linked gene CHD7 in Xenopus laevis embryos disrupted the tadpoles' head structure by decreasing expression of a collagen-producing gene. And in new work, Willsey's group used frogs to show how certain genetic variants can contribute to both autism and congenital heart disease.

Willsey plans to work with Xenopus tropicalis to connect the various biological steps from autism genes to autism traits. "Going from genes to biology to circuits to behavior is a big leap," Willsey says. "One nice thing about frogs is that in one system, you can do all of those things."

Cite this article: https://doi.org/10.53053/WRUR5688 